\title{
Robust Incremental Neural Semantic Graph Parsing
}

\author{
Jan Buys $^{1}$ and Phil Blunsom ${ }^{1,2}$ \\ ${ }^{1}$ Department of Computer Science, University of Oxford $\quad{ }^{2}$ DeepMind \\ \{jan.buys, phil.blunsom\}@cs.ox.ac.uk
}

\begin{abstract}
Parsing sentences to linguisticallyexpressive semantic representations is a key goal of Natural Language Processing. Yet statistical parsing has focussed almost exclusively on bilexical dependencies or domain-specific logical forms. We propose a neural encoder-decoder transition-based parser which is the first full-coverage semantic graph parser for Minimal Recursion Semantics (MRS). The model architecture uses stack-based embedding features, predicting graphs jointly with unlexicalized predicates and their token alignments. Our parser is more accurate than attention-based baselines on MRS, and on an additional Abstract Meaning Representation (AMR) benchmark, and GPU batch processing makes it an order of magnitude faster than a high-precision grammar-based parser. Further, the $86.69 \%$ Smatch score of our MRS parser is higher than the upper-bound on AMR parsing, making MRS an attractive choice as a semantic representation.
\end{abstract}

\section{Introduction}

An important goal of Natural Language Understanding (NLU) is to parse sentences to structured, interpretable meaning representations that can be used for query execution, inference and reasoning. Recently end-to-end models have outperformed traditional pipeline approaches, predicting syntactic or semantic structure as intermediate steps, on NLU tasks such as sentiment analysis and semantic relatedness (Le and Mikolov, 2014; Kiros et al., 2015), question answering (Hermann et al., 2015) and textual entailment (Rocktäschel et al., 2015).
However the linguistic structure used in applications has predominantly been shallow, restricted to bilexical dependencies or trees.

In this paper we focus on robust parsing into linguistically deep representations. The main representation that we use is Minimal Recursion Semantics (MRS) (Copestake et al., 1995, 2005), which serves as the semantic representation of the English Resource Grammar (ERG) (Flickinger, 2000). Existing parsers for full MRS (as opposed to bilexical semantic graphs derived from, but simplifying MRS) are grammar-based, performing disambiguation with a maximum entropy model (Toutanova et al., 2005; Zhang et al., 2007); this approach has high precision but incomplete coverage.

Our main contribution is to develop a fast and robust parser for full MRS-based semantic graphs. We exploit the power of global conditioning enabled by deep learning to predict linguistically deep graphs incrementally. The model does not have access to the underlying ERG or syntactic structures from which the MRS analyses were originally derived. We develop parsers for two graph-based conversions of MRS, Elementary Dependency Structure (EDS) (Oepen and Lønning, 2006) and Dependency MRS (DMRS) (Copestake, 2009), of which the latter is inter-convertible with MRS.

Abstract Meaning Representation (AMR) (Banarescu et al., 2013) is a graph-based semantic representation that shares the goals of MRS. Aside from differences in the choice of which linguistic phenomena are annotated, MRS is a compositional representation explicitly coupled with the syntactic structure of the sentence, while AMR does not assume compositionality or alignment with the sentence structure. Recently a number of AMR parsers have been developed (Flanigan et al., 2014; Wang et al., 2015b; Artzi et al., 2015; 
Damonte et al., 2017), but corpora are still under active development and low inter-annotator agreement places on upper bound of $83 \% \mathrm{~F} 1$ on expected parser performance (Banarescu et al., 2013). We apply our model to AMR parsing by introducing structure that is present explicitly in MRS but not in AMR (Buys and Blunsom, 2017).

Parsers based on RNNs have achieved state-ofthe-art performance for dependency parsing (Dyer et al., 2015; Kiperwasser and Goldberg, 2016) and constituency parsing (Vinyals et al., 2015b; Dyer et al., 2016; Cross and Huang, 2016b). One of the main reasons for the prevalence of bilexical dependencies and tree-based representations is that they can be parsed with efficient and wellunderstood algorithms. However, one of the key advantages of deep learning is the ability to make predictions conditioned on unbounded contexts encoded with RNNs; this enables us to predict more complex structures without increasing algorithmic complexity. In this paper we show how to perform linguistically deep parsing with RNNs.

Our parser is based on a transition system for semantic graphs. However, instead of generating arcs over an ordered, fixed set of nodes (the words in the sentence), we generate the nodes and their alignments jointly with the transition actions. We use a graph-based variant of the arc-eager transition-system. The sentence is encoded with a bidirectional RNN. The transition sequence, seen as a graph linearization, can be predicted with any encoder-decoder model, but we show that using hard attention, predicting the alignments with a pointer network and conditioning explicitly on stack-based features improves performance. In order to deal with data sparsity candidate lemmas are predicted as a pre-processing step, so that the RNN decoder predicts unlexicalized node labels.

We evaluate our parser on DMRS, EDS and AMR graphs. We show that our model architecture improves performance from $79.68 \%$ to $84.16 \%$ F1 over an attention-based encoderdecoder baseline. Although our parser is less accurate that a high-precision grammar-based parser on a test set of sentences parsable by that grammar, incremental prediction and GPU batch processing enables it to parse 529 tokens per second, against 7 tokens per second for the grammarbased parser. On AMR parsing our model obtains $60.11 \%$ Smatch, an improvement of $8 \%$ over an existing neural AMR parser.

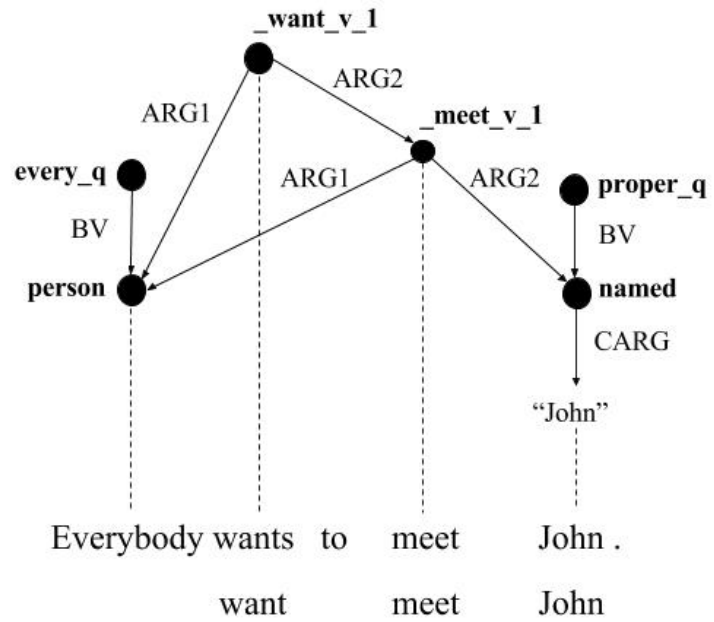

Figure 1: Semantic representation of the sentence "Everybody wants to meet John." The graph is based on the Elementary Dependency Structure (EDS) representation of Minimal Recursion Semantics (MRS). The alignments are given together with the corresponding tokens, and lemmas of surface predicates and constants.

\section{Meaning Representations}

We define a common framework for semantic graphs in which we can place both MRSbased graph representations (DMRS and EDS) and AMR. Sentence meaning is represented with rooted, labelled, connected, directed graphs (Kuhlmann and Oepen, 2016). An example graph is visualized in Figure 1. representations. Node labels are referred to as predicates (concepts in AMR) and edge labels as arguments (AMR relations). In addition constants, a special type of node modifiers, are used to denote the string values of named entities and numbers (including date and time expressions). Every node is aligned to a token or a continuous span of tokens in the sentence the graph corresponds to.

Minimal Recursion Semantics (MRS) is a framework for computational semantics that can be used for parsing or generation (Copestake et al., 2005). Instances and eventualities are represented with logical variables. Predicates take arguments with labels from a small, fixed set of roles. Arguments are either logical variables or handles, designated formalism-internal variables. Handle equality constraints support scope underspecification; multiple scope-resolved logical representations can be derived from one MRS structure. A predicate corresponds to its intrinsic argument 
and is aligned to a character span of the (untokenized) input sentence. Predicates representing named entities or numbers are parameterized by strings. Quantification is expressed through predicates that bound instance variables, rather than through logical operators such as $\exists$ or $\forall$. MRS was designed to be integrated with feature-based grammars such as Head-driven Phrase Structure Grammar (HPSG) (Pollard and Sag, 1994) or Lexical Functional Grammar (LFG) (Kaplan and Bresnan, 1982). MRS has been implement the English Resource Grammar (ERG) (Flickinger, 2000), a broad-coverage high-precision HPSG grammar.

Oepen and Lønning (2006) proposed Elementary Dependency Structure (EDS), a conversion of MRS to variable-free dependency graphs which drops scope underspecification. Copestake (2009) extended this conversion to avoid information loss, primarily through richer edge labels. The resulting representation, Dependency MRS (DMRS), can be converted back to the original MRS, or used directly in MRS-based applications (Copestake et al., 2016). We are interested in the empirical performance of parsers for both of these representations: while EDS is more interpretable as an independent semantic graph representation, DMRS can be related back to underspecified logical forms. A bilexical simplification of EDS has previously been used for semantic dependency parsing (Oepen et al., 2014, 2015). Figure 1 illustrates an EDS graph.

MRS makes an explicit distinction between surface and abstract predicates (by convention surface predicates are prefixed by an underscore). Surface predicates consist of a lemma followed by a coarse part-of-speech tag and an optional sense label. Predicates absent from the ERG lexicon are represented by their surface forms and POS tags. We convert the character-level predicate spans given by MRS to token-level spans for parsing purposes, but the representation does not require gold tokenization. Surface predicates usually align with the span of the token(s) they represent, while abstract predicates can span longer segments. In full MRS every predicate is annotated with a set of morphosyntactic features, encoding for example tense, aspect and number information; we do not currently model these features.

AMR (Banarescu et al., 2013) graphs can be represented in the same framework, despite a number of linguistic differences with MRS. Some in-

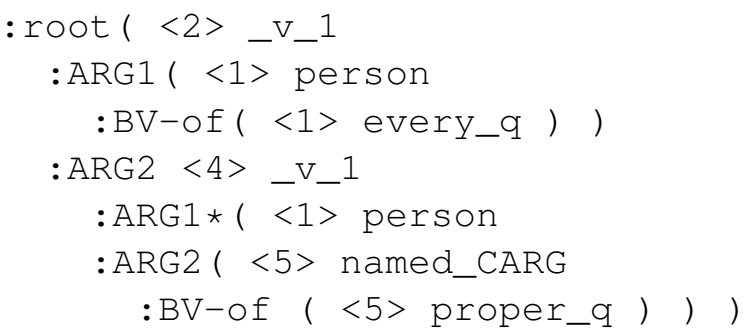

Figure 2: A top-down linearization of the EDS graph in Figure 1, using unlexicalized predicates.

formation annotated explicitly in MRS is latent in AMR, including alignments and the distinction between surface (lexical) and abstract concepts. AMR predicates are based on PropBank (Palmer et al., 2005), annotated as lemmas plus sense labels, but they form only a subset of concepts. Other concepts are either English words or special keywords, corresponding to overt lexemes in some cases but not others.

\section{Incremental Graph Parsing}

We parse sentences to their meaning representations by incrementally predicting semantic graphs together with their alignments. Let $\mathbf{e}=$ $e_{1}, e_{2}, \ldots, e_{I}$ be a tokenized English sentence, $\mathbf{t}=t_{1}, t_{2}, \ldots, t_{J}$ a sequential representation of its graph derivation and $\mathbf{a}=a_{1}, a_{2}, \ldots, a_{J}$ an alignment sequence consisting of integers in the range $1, \ldots, I$. We model the conditional distribution $p(\mathbf{t}, \mathbf{a} \mid \mathbf{e})$ which decomposes as

$$
\prod_{j=1}^{J} p\left(a_{j} \mid(\mathbf{a}, \mathbf{t})_{1: j-1}, \mathbf{e}\right) p\left(t_{j} \mid \mathbf{a}_{1: j}, \mathbf{t}_{1: j-1}, \mathbf{e}\right) .
$$

We also predict the end-of-span alignments as a seperate sequence $\mathbf{a}^{(\mathbf{e})}$.

\subsection{Top-down linearization}

We now consider how to linearize the semantic graphs, before defining the neural models to parameterize the parser in section 4 . The first approach is to linearize a graph as the pre-order traversal of its spanning tree, starting at a designated root node (see Figure 2). Variants of this approach have been proposed for neural constituency parsing (Vinyals et al., 2015b), logical form prediction (Dong and Lapata, 2016; Jia and Liang, 2016) and AMR parsing (Barzdins and Gosko, 2016; Peng et al., 2017).

In the linearization, labels of edges whose direction are reversed in the spanning tree are marked 
by adding - of. Edges not included in the spanning tree, referred to as reentrancies, are represented with special edges whose dependents are dummy nodes pointing back to the original nodes. Our potentially lossy representation represents these edges by repeating the dependent node labels and alignments, which are recovered heuristically. The alignment does not influence the linearized node ordering.

\subsection{Transition-based parsing}

Figure 1 shows that the semantic graphs we work with can also be interpreted as dependency graphs, as nodes are aligned to sentence tokens. Transition-based parsing (Nivre, 2008) has been used extensively to predict dependency graphs incrementally. We apply a variant of the arc-eager transition system that has been proposed for graph (as opposed to tree) parsing (Sagae and Tsujii, 2008; Titov et al., 2009; Gómez-Rodríguez and Nivre, 2010) to derive a transition-based parser for deep semantic graphs. In dependency parsing the sentence tokens also act as nodes in the graph, but here we need to generate the nodes incrementally as the transition-system proceeds, conditioning the generation on the given sentence. Damonte et al. (2017) proposed an arc-eager AMR parser, but their transition system is more narrowly restricted to AMR graphs.

The transition system consists of a stack of graph nodes being processed and a buffer, holding a single node at a time. The main transition actions are shift, reduce, left-arc, right-arc. Figure 3 shows an example transition sequence together with the stack and buffer after each step. The shift transition moves the element on the buffer to the top of the stack, and generates a predicate and its alignment as the next node on the buffer. Left-arc and right-arc actions add labeled arcs between the buffer and stack top (for DMRS a transition for undirected arcs is included), but do not change the state of the stack or buffer. Finally, reduce pops the top element from the stack, and predicts its end-ofspan alignment (if included in the representation). To predict non-planar arcs, we add another transition, which we call cross-arc, which first predicts the stack index of a node which is not on top of the stack, adding an arc between the head of the buffer and that node. Another special transition designates the buffer node as the root.

To derive an oracle for this transition system, it is necessary to determine the order in which the nodes are generated. We consider two approaches. The first ordering is obtained by performing an in-order traversal of the spanning tree, where the node order is determined by the alignment. In the resulting linearization the only non-planar arcs are reentrancies. The second approach lets the ordering be monotone (non-decreasing) with respect to the alignments, while respecting the in-order ordering for nodes with the same alignment. In an arc-eager oracle arcs are added greedily, while a reduce action can either be performed as soon as the stack top node has been connected to all its dependents, or delayed until it has to reduce to allow the correct parse tree to be formed. In our model the oracle delays reduce, where possible, until the end alignment of the stack top node spans the node on the buffer. As the span end alignments often cover phrases that they head (e.g. for quantifiers) this gives a natural interpretation to predicting the span end together with the reduce action.

\subsection{Delexicalization and lemma prediction}

Each token in MRS annotations is aligned to at most one surface predicate. We decompose surface predicate prediction by predicting candidate lemmas for input tokens, and delexicalized predicates consisting only of sense labels. The full surface predicates are then recovered through the predicted alignments.

We extract a dictionary mapping words to lemmas from the ERG lexicon. Candidate lemmas are predicted using this dictionary, and where no dictionary entry is available with a lemmatizer. The same approach is applied to predict constants, along with additional normalizations such as mapping numbers to digit strings.

We use the Stanford CoreNLP toolkit (Manning et al., 2014) to tokenize and lemmatize sentences, and tag tokens with the Stanford Named Entity Recognizer (Finkel et al., 2005). The tokenization is customized to correspond closely to the ERG tokenization; hyphens are removed pre-processing step. For AMR we use automatic alignments and the graph topology to classify concepts as surface or abstract (Buys and Blunsom, 2017). The lexicon is restricted to Propbank (Palmer et al., 2005) predicates; for other concepts we extract a lexicon from the training data. 


\begin{tabular}{|c|c|c|c|}
\hline Action & Stack & Buffer & Arc added \\
\hline init(1, person) & [] & $(1,1$, person $)$ & - \\
\hline $\operatorname{sh}(1$, & {$[(1$,} & $(2,1$, & - \\
\hline la $(B$ & {$[(1,1$, person $)]$} & $(2,1$, every_q) & $(2, \mathrm{BV}, 1)$ \\
\hline $\operatorname{sh}\left(2,{ }_{-} \_1\right)$ & {$[(1]$,} & $\left(2,1,{ }_{-} \_1\right)$ & - \\
\hline re & {$[(1$,} & $\left(3,2, \mathrm{v}_{-} 1\right)$ & 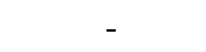 \\
\hline la(ARG1) & {$[(1,1$, person $)]$} & $\left(3,2, v_{-} 1\right)$ & $(3$, ARG1, 1) \\
\hline
\end{tabular}

Figure 3: Start of the transition sequence for parsing the graph in Figure 1. The transitions are shift $(\mathrm{sh})$, reduce (re), left arc (la) and right arc ( $\mathrm{ra}$ ). The action taken at each step is given, along with the state of the stack and buffer after the action is applied, and any arcs added. Shift transitions generate the alignments and predicates of the nodes placed on the buffer. Items on the stack and buffer have the form (node index, alignment, predicate label), and arcs are of the form (head index, argument label, dependent index).

\section{Encoder-Decoder Models}

\subsection{Sentence encoder}

The sentence $\mathbf{e}$ is encoded with a bidirectional RNN. We use a standard LSTM architecture without peephole connections (Jozefowicz et al., 2015). For every token $e$ we embed its word, POS tag and named entity (NE) tag as vectors $x_{w}, x_{t}$ and $x_{n}$, respectively.

The embeddings are concatenated and passed through a linear transformation

$$
g(e)=W^{(x)}\left[x_{w} ; x_{t} ; x_{n}\right]+b^{x},
$$

such that $g(e)$ has the same dimension as the LSTM. Each input position $i$ is represented by a hidden state $h_{i}$, which is the concatenation of its forward and backward LSTM state vectors.

\subsection{Hard attention decoder}

We model the alignment of graph nodes to sentence tokens, a, as a random variable. For the arceager model, $a_{j}$ corresponds to the alignment of the node of the buffer after action $t_{j}$ is executed. The distribution of $t_{j}$ is over all transitions and predicates (corresponding to shift transitions), predicted with a single softmax.

The parser output is predicted by an RNN decoder. Let $s_{j}$ be the decoder hidden state at output position $j$. We initialize $s_{0}$ with the final state of the backward encoder. The alignment is predicted with a pointer network (Vinyals et al., 2015a).

The logits are computed with an MLP scoring the decoder hidden state against each of the encoder hidden states (for $i=1, \ldots, I$ ),

$$
u_{j}^{i}=w^{T} \tanh \left(W^{(1)} h_{i}+W^{(2)} s_{j}\right) .
$$

The alignment distribution is then estimated by

$$
p\left(a_{j}=i \mid \mathbf{a}_{1: j-1}, \mathbf{t}_{1: j-1}, \mathbf{e}\right)=\operatorname{softmax}\left(u_{j}^{i}\right) .
$$

To predict the next transition $t_{i}$, the output vector is conditioned on the encoder state vector $h_{a_{j}}$, corresponding to the alignment:

$$
\begin{aligned}
& o_{j}=W^{(3)} s_{j}+W^{(4)} h_{a_{j}} \\
& v_{j}=R^{(d)} o_{j}+b^{(d)},
\end{aligned}
$$

where $R^{(d)}$ and $b^{(d)}$ are the output representation matrix and bias vector, respectively.

The transition distribution is then given by

$$
p\left(t_{j} \mid \mathbf{a}_{1: j}, \mathbf{t}_{1: j-1}, \mathbf{e}\right)=\operatorname{softmax}\left(v_{j}\right) .
$$

Let $e(t)$ be the embedding of decoder symbol $t$. The RNN state at the next time-step is computed as

$$
\begin{aligned}
d_{j+1} & =W^{(5)} e\left(t_{j}\right)+W^{(6)} h_{a_{j}} \\
s_{j+1} & =R N N\left(d_{j+1}, s_{j}\right) .
\end{aligned}
$$

The end-of-span alignment $a_{j}^{(e)}$ for MRS-based graphs is predicted with another pointer network. The end alignment of a token is predicted only when a node is reduced from the stack, therefore this alignment is not observed at each time-step; it is also not fed back into the model.

The hard attention approach, based on supervised alignments, can be contrasted to soft attention, which learns to attend over the input without supervision. The attention is computed as with hard attention, as $\alpha_{j}^{i}=\operatorname{softmax}\left(u_{j}^{i}\right)$. However instead of making a hard selection, a weighted average over the encoder vectors is computed as $q_{j}=\sum_{i=1}^{i=I} \alpha_{j}^{i} h_{i}$. This vector is used instead of $h_{a_{j}}$ for prediction and feeding to the next timestep. 


\subsection{Stack-based model}

We extend the hard attention model to include features based on the transition system stack. These features are embeddings from the bidirectional RNN encoder, corresponding to the alignments of the nodes on the buffer and on top of the stack. This approach is similar to the features proposed by Kiperwasser and Goldberg (2016) and Cross and Huang (2016a) for dependency parsing, although they do not use RNN decoders.

To implement these features the layer that computes the output vector is extended to

$$
o_{j}=W^{(3)} s_{j}+W^{(4)} h_{a_{j}}+W^{(7)} h_{\mathrm{st}_{0}},
$$

where $s t_{0}$ is the sentence alignment index of the element on top of the stack. The input layer to the next RNN time-step is similarly extended to

$$
d_{j+1}=W^{(5)} e\left(t_{j}\right)+W^{(6)} h_{\mathrm{buf}}+W^{(8)} h_{\mathrm{st} 0},
$$

where buf is the buffer alignment after $t_{j}$ is executed.

Our implementation of the stack-based model enables batch processing in static computation graphs, similar to Bowman et al. (2016). We maintain a stack of alignment indexes for each element in the batch, which is updated inside the computation graph after each parsing action. This enables minibatch SGD during training as well as efficient batch decoding.

We perform greedy decoding. For the stackbased model we ensure that if the stack is empty, the next transition predicted has to be shift. For the other models we ensure that the output is wellformed during post-processing by robustly skipping over out-of-place symbols or inserting missing ones.

\section{Related Work}

Prior work for MRS parsing predominantly predicts structures in the context of grammar-based parsing, where sentences are parsed to HPSG derivations consistent with the grammar, in this case the ERG (Flickinger, 2000). The nodes in the derivation trees are feature structures, from which MRS is extracted through unification. This approach fails to parse sentences for which no valid derivation is found. Maximum entropy models are used to score the derivations in order to find the most likely parse (Toutanova et al., 2005). This approach is implemented in the PET (Callmeier, 2000) and $\mathrm{ACE}^{1}$ parsers.

There have also been some efforts to develop robust MRS parsers. One proposed approach learns a PCFG grammar to approximate the HPSG derivations (Zhang and Krieger, 2011; Zhang et al., 2014). MRS is then extracted with robust unification to compose potentially incompatible feature structures, although that still fails for a small proportion of sentences. The model is trained on a large corpus of Wikipedia text parsed with the grammar-based parser. Ytrest $\varnothing 1$ (2012) proposed a transition-based approach to HPSG parsing that produces derivations from which both syntactic and semantic (MRS) parses can be extracted. The parser has an option not to be restricted by the ERG. However, neither of these approaches have results available that can be compared directly to our setup, or generally available implementations.

Although AMR parsers produce graphs that are similar in structure to MRS-based graphs, most of them make assumptions that are invalid for MRS, and rely on extensive external AMR-specific resources. Flanigan et al. (2014) proposed a twostage parser that first predicts concepts or subgraphs corresponding to sentence segments, and then parses these concepts into a graph structure. However MRS has a large proportion of abstract nodes that cannot be predicted from short segments, and interact closely with the graph structure. Wang et al. $(2015 \mathrm{~b}, \mathrm{a})$ proposed a custom transition-system for AMR parsing that converts dependency trees to AMR graphs, relying on assumptions on the relationship between these. Pust et al. (2015) proposed a parser based on syntaxbased machine translation (MT), while AMR has also been integrated into CCG Semantic Parsing (Artzi et al., 2015; Misra and Artzi, 2016). Recently Damonte et al. (2017) and Peng et al. (2017) proposed AMR parsers based on neural networks.

\section{Experiments}

\subsection{Data}

DeepBank (Flickinger et al., 2012) is an HPSG and MRS annotation of the Penn Treebank Wall Street Journal (WSJ) corpus. It was developed following an approach known as dynamic treebanking (Oepen et al., 2004) that couples treebank annotation with grammar development, in this case

\footnotetext{
${ }^{1}$ http://sweaglesw.org/linguistics/ace/
} 
of the ERG. This approach has been shown to lead to high inter-annotator agreement: 0.94 against 0.71 for AMR (Bender et al., 2015). Parses are only provided for sentences for which the ERG has an analysis acceptable to the annotator - this means that we cannot evaluate parsing accuracy for sentences which the ERG cannot parse (approximately $15 \%$ of the original corpus).

We use Deepbank version 1.1, corresponding to ERG $1214^{2}$, following the suggested split of sections 0 to 19 as training data data, 20 for development and 21 for testing. The gold-annotated training data consists of 35,315 sentences. We use the LOGON environment ${ }^{3}$ and the pyDelphin library ${ }^{4}$ to extract DMRS and EDS graphs.

For AMR parsing we use LDC2015E86, the dataset released for the SemEval 2016 AMR parsing Shared Task (May, 2016). This data includes newswire, weblog and discussion forum text. The training set has 16,144 sentences. We obtain alignments using the rule-based JAMR aligner (Flanigan et al., 2014).

\subsection{Evaluation}

Dridan and Oepen (2011) proposed an evaluation metric called Elementary Dependency Matching (EDM) for MRS-based graphs. EDM computes the F1-score of tuples of predicates and arguments. A predicate tuple consists of the label and character span of a predicate, while an argument tuple consists of the character spans of the head and dependent nodes of the relation, together with the argument label. In order to tolerate subtle tokenization differences with respect to punctuation, we allow span pairs whose ends differ by one character to be matched.

The Smatch metric (Cai and Knight, 2013), proposed for evaluating AMR graphs, also measures graph overlap, but does not rely on sentence alignments to determine the correspondences between graph nodes. Smatch is instead computed by performing inference over graph alignments to estimate the maximum F1-score obtainable from a one-to-one matching between the predicted and gold graph nodes.

\footnotetext{
${ }^{2}$ http://svn.delph-in.net/erg/tags/ $1214 /$

${ }^{3}$ http://moin. delph-in. net/LogonTop

${ }^{4}$ https://github.com/delph-in/pydelphin
}

\begin{tabular}{l|c|c|c} 
Model & EDM & $\mathrm{EDM}_{P}$ & $\mathrm{EDM}_{A}$ \\
\hline TD lex & 81.44 & 85.20 & 76.87 \\
TD unlex & 81.72 & 85.59 & 77.04 \\
AE lex & 81.35 & 85.79 & 76.02 \\
AE unlex & 82.56 & 86.76 & 77.54
\end{tabular}

Table 1: DMRS development set results for attention-based encoder-decoder models with alignments encoded in the linearization, for topdown (TD) and arc-eager (AE) linearizations, and lexicalized and unlexicalized predicate prediction.

\subsection{Model setup}

Our parser ${ }^{5}$ is implemented in TensorFlow (Abadi et al., 2015). For training we use Adam (Kingma and $\mathrm{Ba}, 2015)$ with learning rate 0.01 and batchsize 64. Gradients norms are clipped to 5.0 (Pascanu et al., 2013). We use single-layer LSTMs with dropout of 0.3 (tuned on the development set) on input and output connections. We use encoder and decoder embeddings of size 256, and POS and NE tag embeddings of size 32, For DMRS and EDS graphs the hidden units size is set to 256 , for AMR it is 128. This configuration, found using grid search and heuristic search within the range of models that fit into a single GPU, gave the best performance on the development set under multiple graph linearizations. Encoder word embeddings are initialized (in the first 100 dimensions) with pre-trained order-sensitive embeddings (Ling et al., 2015). Singletons in the encoder input are replaced with an unknown word symbol with probability 0.5 for each iteration.

\subsection{MRS parsing results}

We compare different linearizations and model architectures for parsing DMRS on the development data, showing that our approach is more accurate than baseline neural approaches. We report EDM scores, including scores for predicate $\left(\mathrm{EDM}_{P}\right)$ and argument $\left(\mathrm{EDM}_{A}\right)$ prediction.

First we report results using standard attentionbased encoder-decoders, with the alignments encoded as token strings in the linearization. (Table 1). We compare the top-down (TD) and arceager (AE) linearizations, as well as the effect of delexicalizing the predicates (factorizing lemmas out of the linearization and predicting them sepa-

\footnotetext{
${ }^{5}$ Code and data preparation scripts are available at https://github.com/janmbuys/ DeepDeepParser.
} 


\begin{tabular}{l|c|c|c} 
Model & EDM & EDM $_{P}$ & $\mathrm{EDM}_{A}$ \\
\hline TD soft & 81.53 & 85.32 & 76.94 \\
TD hard & 82.75 & 86.37 & 78.37 \\
AE hard & 84.65 & 87.77 & 80.85 \\
AE stack & 85.28 & 88.38 & 81.51
\end{tabular}

Table 2: DMRS development set results of encoder-decoder models with pointer-based alignment prediction, delexicalized predicates and hard or soft attention.

rately.) In both cases constants are predicted with a dictionary lookup based on the predicted spans. A special label is predicted for predicates not in the ERG lexicon - the words and POS tags that make up those predicates are recovered through the alignments during post-processing.

The arc-eager unlexicalized representation gives the best performance, even though the model has to learn to model the transition system stack through the recurrent hidden states without any supervision of the transition semantics. The unlexicalized models are more accurate, mostly due to their ability to generalize to sparse or unseen predicates occurring in the lexicon. For the arc-eager representation, the oracle EDM is $99 \%$ for the lexicalized representation and $98.06 \%$ for the delexicalized representation. The remaining errors are mostly due to discrepancies between the tokenization used by our system and the ERG tokenization. The unlexicalized models are also faster to train, as the decoder's output vocabulary is much smaller, reducing the expense of computing softmaxes over large vocabularies.

Next we consider models with delexicalized linearizations that predict the alignments with pointer networks, contrasting soft and hard attention models (Table 2). The results show that the arc-eager models performs better than those based on topdown representation. For the arc-eager model we use hard attention, due to the natural interpretation of the alignment prediction corresponding to the transition system. The stack-based architecture gives further improvements.

When comparing the effect of different predicate orderings for the arc-eager model, we find that the monotone ordering performs $0.44 \mathrm{EDM}$ better than the in-order ordering, despite having to parse more non-planar dependencies.

We also trained models that only predict predicates (in monotone order) together with their

\begin{tabular}{l|c|c|c} 
Model & TD RNN & AE RNN & ACE \\
\hline EDM & 79.68 & 84.16 & 89.64 \\
$\mathrm{EDM}_{P}$ & 83.36 & 87.54 & 92.08 \\
$\mathrm{EDM}_{A}$ & 75.16 & 80.10 & 86.77 \\
Start EDM & 84.44 & 87.81 & 91.91 \\
Start EDM & 80.93 & 85.61 & 89.28 \\
Smatch & 85.28 & 86.69 & 93.50
\end{tabular}

Table 3: DMRS parsing test set results, comparing the standard top-down attention-based and arceager stack-based RNN models to the grammarbased ACE parser.

start spans. The hard attention model obtains $91.36 \% \mathrm{~F} 1$ on predicates together with their start spans with the unlexicalized model, compared to $88.22 \%$ for lexicalized predicates and $91.65 \%$ for the full parsing model.

Table 3 reports test set results for various evaluation metrics. Start EDM is calculated by requiring only the start of the alignment spans to match, not the ends. We compare the performance of our baseline and stack-based models against ACE, the ERG-based parser.

Despite the promising performance of the model a gap remains between the accuracy of our parser and ACE. One reason for this is that the test set sentences will arguably be easier for ACE to parse as their choice was restricted by the same grammar that ACE uses. EDM metrics excluding end-span prediction (Start EDM) show that our parser has relatively more difficulty in parsing end-span predictions than the grammar-based parser.

We also evaluate the speed of our model compared with ACE. For the unbatched version of our model, the stack-based parser parses 41.63 tokens per second, while the batched implementation parses 529.42 tokens per second using a batch size of 128. In comparison, the setting of ACE for which we report accuracies parses 7.47 tokens per second. By restricting the memory usage of ACE, which restricts its coverage, we see that ACE can parse 11.07 tokens per second at $87.7 \%$ coverage, and 15.11 tokens per second at $77.8 \%$ coverage.

Finally we report results for parsing EDS (Table 4). The EDS parsing task is slightly simpler than DMRS, due to the absence of rich argument labels and additional graph edges that allow the recovery of full MRS. We see that for ACE the accuracies are very similar, while for our model EDS 


\begin{tabular}{l|c|c} 
Model & AE RNN & ACE \\
\hline EDM & 85.48 & 89.58 \\
EDM $_{P}$ & 88.14 & 91.82 \\
EDM $_{A}$ & 82.20 & 86.92 \\
Smatch & 86.50 & 93.52
\end{tabular}

Table 4: EDS parsing test set results.

\begin{tabular}{l|c|c} 
Model & Concept F1 & Smatch \\
\hline TD no pointers & 70.16 & 57.95 \\
TD soft & 71.25 & 59.39 \\
TD soft unlex & 72.62 & 59.88 \\
AE hard unlex & 76.83 & 59.83 \\
AE stack unlex & 77.93 & 61.21
\end{tabular}

Table 5: Development set results for AMR parsing. All the models except the first predict alignments with pointer networks.

parsing is more accurate on the EDM metrics. We hypothesize that most of the extra information in DMRS can be obtained through the ERG, to which ACE has access but our model doesn't.

An EDS corpus which consists of about $95 \%$ of the DeepBank data has also been released ${ }^{6}$, with the goal of enabling comparison with other semantic graph parsing formalisms, including CCG dependencies and Prague Semantic Dependencies, on the same data set (Kuhlmann and Oepen, 2016). On this corpus our model obtains 85.87 EDM and 85.49 Smatch.

\subsection{AMR parsing}

We apply the same approach to AMR parsing. Results on the development set are given in Table 5. The arc-eager-based models again give better performance, mainly due to improved concept prediction accuracy. However, concept prediction remains the most important weakness of the model; Damonte et al. (2017) reports that state-of-the-art AMR parsers score $83 \%$ on concept prediction.

We report test set results in Table 6. Our best neural model outperforms the baseline JAMR parser (Flanigan et al., 2014), but still lags behind the performance of state-of-the-art AMR parsers such as CAMR (Wang et al., 2016) and AMR Eager (Damonte et al., 2017). These models make extensive use of external resources, including syntactic parsers and semantic role labellers. Our attention-based encoder-decoder model already outperforms previous sequence-to-sequence

\footnotetext{
${ }^{6}$ http: //sdp.delph-in.net/osdp-12.tgz
}

\begin{tabular}{l|c} 
Model & Smatch \\
\hline Flanigan et al. (2014) & 56 \\
\hline Wang et al. (2016) & 66.54 \\
Damonte et al. (2017) & 64 \\
\hline Peng and Gildea (2016) & 55 \\
Peng et al. (2017) & 52 \\
Barzdins and Gosko (2016) & 43.3 \\
\hline TD no pointers & 56.56 \\
AE stack delex & 60.11
\end{tabular}

Table 6: AMR parsing test set results (Smatch F1 scores). Published results follow the number of decimals which were reported.

AMR parsers (Barzdins and Gosko, 2016; Peng et al., 2017), and the arc-eager model boosts accuracy further. Our model also outperforms a Synchronous Hyperedge Replacement Grammar model (Peng and Gildea, 2016) which is comparable as it does not make extensive use of external resources.

\section{Conclusion}

In this paper we advance the state of parsing by employing deep learning techniques to parse sentence to linguistically expressive semantic representations that have not previously been parsed in an end-to-end fashion. We presented a robust, wide-coverage parser for MRS that is faster than existing parsers and amenable to batch processing. We believe that there are many future avenues to explore to further increase the accuracy of such parsers, including different training objectives, more structured architectures and semisupervised learning.

\section{Acknowledgments}

The first author thanks the financial support of the Clarendon Fund and the Skye Foundation. We thank Stephan Oepen for feedback and help with data preperation, and members of the Oxford NLP group for valuable discussions.

\section{References}

Martín Abadi, Ashish Agarwal, Paul Barham, Eugene Brevdo, Zhifeng Chen, Craig Citro, Greg S. Corrado, Andy Davis, Jeffrey Dean, Matthieu Devin, Sanjay Ghemawat, Ian Goodfellow, Andrew Harp, Geoffrey Irving, Michael Isard, Yangqing Jia, Rafal Jozefowicz, Lukasz Kaiser, Manjunath Kudlur, Josh Levenberg, Dan Mané, Rajat Monga, Sherry Moore, 
Derek Murray, Chris Olah, Mike Schuster, Jonathon Shlens, Benoit Steiner, Ilya Sutskever, Kunal Talwar, Paul Tucker, Vincent Vanhoucke, Vijay Vasudevan, Fernanda Viégas, Oriol Vinyals, Pete Warden, Martin Wattenberg, Martin Wicke, Yuan Yu, and Xiaoqiang Zheng. 2015. TensorFlow: Large-scale machine learning on heterogeneous systems. Software available from tensorflow.org. http://tensorflow.org/.

Yoav Artzi, Kenton Lee, and Luke Zettlemoyer. 2015. Broad-coverage CCG semantic parsing with AMR. In Proceedings of the 2015 Conference on Empirical Methods in Natural Language Processing. Association for Computational Linguistics, Lisbon, Portugal, pages 1699-1710. http://aclweb.org/anthology/D15-1198.

Laura Banarescu, Claire Bonial, Shu Cai, Madalina Georgescu, Kira Griffitt, Ulf Hermjakob, Kevin Knight, Philipp Koehn, Martha Palmer, and Nathan Schneider. 2013. Abstract meaning representation for sembanking. In Proceedings of the 7th Linguistic Annotation Workshop and Interoperability with Discourse. Association for Computational Linguistics, Sofia, Bulgaria, pages 178-186. http://www.aclweb.org/anthology/W13-2322.

Guntis Barzdins and Didzis Gosko. 2016. Riga at semeval-2016 task 8: Impact of smatch extensions and character-level neural translation on AMR parsing accuracy. In Proceedings of SemEval.

Emily M Bender, Dan Flickinger, Stephan Oepen, Woodley Packard, and Ann Copestake. 2015. Layers of interpretation: On grammar and compositionality. In Proceedings of the 11th International Conference on Computational Semantics. pages 239249.

Samuel R. Bowman, Jon Gauthier, Abhinav Rastogi, Raghav Gupta, Christopher D. Manning, and Christopher Potts. 2016. A fast unified model for parsing and sentence understanding. In Proceedings of ACL. pages 1466-1477. http://www.aclweb.org/anthology/P16-1139.

Jan Buys and Phil Blunsom. 2017. Oxford at SemEval2017 Task 9: Neural AMR parsing with pointeraugmented attention. In Proceedings of SemEval.

Shu Cai and Kevin Knight. 2013. Smatch: An evaluation metric for semantic feature structures. In Proceedings of ACL (short papers).

Ulrich Callmeier. 2000. PET - a platform for experimentation with efficient HPSG processing techniques. Natural Language Engineering 6(1):99107.

Ann Copestake. 2009. Invited talk: Slacker semantics: Why superficiality, dependency and avoidance of commitment can be the right way to go. In Proceedings of EACL. pages 1-9. http://www.aclweb.org/anthology/E09-1001.
Ann Copestake, Guy Emerson, Michael Wayne Goodman, Matic Horvat, Alexander Kuhnle, and Ewa Muszyska. 2016. Resources for building applications with dependency minimal recursion semantics. In Proceedings of the Tenth International Conference on Language Resources and Evaluation (LREC 2016).

Ann Copestake, Dan Flickinger, Rob Malouf, Susanne Riehemann, and Ivan Sag. 1995. Translation using minimal recursion semantics. In In Proceedings of the Sixth International Conference on Theoretical and Methodological Issues in Machine Translation.

Ann Copestake, Dan Flickinger, Carl Pollard, and Ivan A Sag. 2005. Minimal recursion semantics: An introduction. Research on Language and Computation 3(2-3):281-332.

James Cross and Liang Huang. 2016a. Incremental parsing with minimal features using bi-directional lstm. In Proceedings of ACL. page 32.

James Cross and Liang Huang. 2016b. Spanbased constituency parsing with a structurelabel system and provably optimal dynamic oracles. In Proceedings of EMNLP. pages 1-11. https://aclweb.org/anthology/D16-1001.

Marco Damonte, Shay B. Cohen, and Giorgio Satta. 2017. An incremental parser for abstract meaning representation. In Proceedings of EACL. pages 536546. http://www.aclweb.org/anthology/E17-1051.

Li Dong and Mirella Lapata. 2016. Language to logical form with neural attention. In Proceedings of ACL. pages 33-43. http://www.aclweb.org/anthology/P16-1004.

Rebecca Dridan and Stephan Oepen. 2011. Parser evaluation using elementary dependency matching. In Proceedings of the 12th International Conference on Parsing Technologies. Association for Computational Linguistics, pages 225-230.

Chris Dyer, Miguel Ballesteros, Wang Ling, Austin Matthews, and Noah A. Smith. 2015. Transitionbased dependency parsing with stack long shortterm memory. In Proceedings of ACL. pages 334343. http://www.aclweb.org/anthology/P15-1033.

Chris Dyer, Adhiguna Kuncoro, Miguel Ballesteros, and Noah A. Smith. 2016. Recurrent neural network grammars. In Proceedings of NAACL.

Jenny Rose Finkel, Trond Grenager, and Christopher Manning. 2005. Incorporating non-local information into information extraction systems by Gibbs sampling. In Proceedings of ACL. pages 363-370. http://dx.doi.org/10.3115/1219840.1219885.

Jeffrey Flanigan, Sam Thomson, Jaime G. Carbonell, Chris Dyer, and Noah A. Smith. 2014. A discriminative graph-based parser for the abstract meaning representation. In Proceedings of ACL. pages 14261436. http://aclweb.org/anthology/P/P14/P141134.pdf. 
Dan Flickinger. 2000. On building a more effcient grammar by exploiting types. Natural Language Engineering 6(01):15-28.

Dan Flickinger, Yi Zhang, and Valia Kordoni. 2012. Deepbank. a dynamically annotated treebank of the wall street journal. In Proceedings of the 11th International Workshop on Treebanks and Linguistic Theories. pages 85-96.

Carlos Gómez-Rodríguez and Joakim Nivre. 2010. A transition-based parser for 2-planar dependency structures. In Proceedings of ACL. pages 14921501. http://www.aclweb.org/anthology/P10-1151.

Karl Moritz Hermann, Tomas Kocisky, Edward Grefenstette, Lasse Espeholt, Will Kay, Mustafa Suleyman, and Phil Blunsom. 2015. Teaching machines to read and comprehend. In Advances in Neural Information Processing Systems. pages 16931701.

Robin Jia and Percy Liang. 2016. Data recombination for neural semantic parsing. In Proceedings of ACL. pages 12-22. http://www.aclweb.org/anthology/P16-1002.

Rafal Jozefowicz, Wojciech Zaremba, and Ilya Sutskever. 2015. An empirical exploration of recurrent network architectures. In Proceedings of ICML. pages 2342-2350.

Ronald M Kaplan and Joan Bresnan. 1982. Lexicalfunctional grammar: A formal system for grammatical representation. Formal Issues in LexicalFunctional Grammar pages 29-130.

Diederik P. Kingma and Jimmy Ba. 2015. Adam: A method for stochastic optimization. In Proceedings of ICLR. http://arxiv.org/abs/1412.6980.

Eliyahu Kiperwasser and Yoav Goldberg. 2016. Simple and accurate dependency parsing using bidirectional lstm feature representations. Transactions of the Association for Computational Linguistics 4:313-327.

Ryan Kiros, Yukun Zhu, Ruslan R Salakhutdinov, Richard Zemel, Raquel Urtasun, Antonio Torralba, and Sanja Fidler. 2015. Skip-thought vectors. In Advances in neural information processing systems. pages 3294-3302.

Marco Kuhlmann and Stephan Oepen. 2016. Towards a catalogue of linguistic graph banks. Computational Linguistics 42(4):819-827.

Quoc V Le and Tomas Mikolov. 2014. Distributed representations of sentences and documents. In ICML. volume 14, pages 1188-1196.

Wang Ling, Chris Dyer, Alan W Black, and Isabel Trancoso. 2015. Two/too simple adaptations of word2vec for syntax problems. In Proceedings of NAACL-HLT. pages 1299-1304. http://www.aclweb.org/anthology/N15-1142.
Christopher D. Manning, Mihai Surdeanu, John Bauer, Jenny Finkel, Steven J. Bethard, and David McClosky. 2014. The Stanford CoreNLP natural language processing toolkit. In ACL System Demonstrations. pages 55-60. http://www.aclweb.org/anthology/P/P14/P14-5010.

Jonathan May. 2016. Semeval-2016 task 8: Meaning representation parsing. In Proceedings of SemEval. pages 1063-1073. http://www.aclweb.org/anthology/S16-1166.

Dipendra Kumar Misra and Yoav Artzi. 2016. Neural shift-reduce ccg semantic parsing. In Proceedings of EMNLP. Austin, Texas, pages 1775-1786. https://aclweb.org/anthology/D16-1183.

Joakim Nivre. 2008. Algorithms for deterministic incremental dependency parsing. Computational Linguistics 34(4):513-553.

Stephan Oepen, Dan Flickinger, Kristina Toutanova, and Christopher D. Manning. 2004. Lingo redwoods. Research on Language and Computation 2(4):575-596. https://doi.org/10.1007/s11168-0047430-4.

Stephan Oepen, Marco Kuhlmann, Yusuke Miyao, Daniel Zeman, Silvie Cinkova, Dan Flickinger, Jan Hajic, and Zdenka Uresova. 2015. Semeval 2015 task 18: Broad-coverage semantic dependency parsing. In Proceedings of SemEval. pages 915-926. http://www.aclweb.org/anthology/S15-2153.

Stephan Oepen, Marco Kuhlmann, Yusuke Miyao, Daniel Zeman, Dan Flickinger, Jan Hajic, Angelina Ivanova, and Yi Zhang. 2014. Semeval 2014 task 8: Broad-coverage semantic dependency parsing. In Proceedings of SemEval. pages 63-72. http://www.aclweb.org/anthology/S14-2008.

Stephan Oepen and Jan Tore Lønning. 2006. Discriminant-based MRS banking. In Proceedings of the 5th International Conference on Language Resources and Evaluation. pages 1250-1255.

Martha Palmer, Daniel Gildea, and Paul Kingsbury. 2005. The proposition bank: An annotated corpus of semantic roles. Computational linguistics 31(1):71106.

Razvan Pascanu, Tomas Mikolov, and Yoshua Bengio. 2013. On the difficulty of training recurrent neural networks. ICML (3) 28:1310-1318.

Xiaochang Peng and Daniel Gildea. 2016. Uofr at semeval-2016 task 8: Learning synchronous hyperedge replacement grammar for amr parsing. In Proceedings of SemEval-2016. pages 1185-1189. http://www.aclweb.org/anthology/S16-1183.

Xiaochang Peng, Chuan Wang, Daniel Gildea, and Nianwen Xue. 2017. Addressing the data sparsity issue in neural amr parsing. In Proceedings of EACL. Preprint. http://www.cs.brandeis.edu/ cwang24/files/eacl17.pdf. 
Carl Pollard and Ivan A Sag. 1994. Head-driven phrase structure grammar. University of Chicago Press.

Michael Pust, Ulf Hermjakob, Kevin Knight, Daniel Marcu, and Jonathan May. 2015. Parsing English into abstract meaning representation using syntax-based machine translation. In Proceedings of EMNLP. Association for Computational Linguistics, Lisbon, Portugal, pages 1143-1154. http://aclweb.org/anthology/D15-1136.

Tim Rocktäschel, Edward Grefenstette, Karl Moritz Hermann, Tomáš Kočiskỳ, and Phil Blunsom. 2015. Reasoning about entailment with neural attention. arXiv preprint arXiv:1509.06664 .

Kenji Sagae and Jun'ichi Tsujii. 2008. Shiftreduce dependency DAG parsing. In Proceedings of Coling 2008. pages 753-760. http://www.aclweb.org/anthology/C08-1095.

Ivan Titov, James Henderson, Paola Merlo, and Gabriele Musillo. 2009. Online graph planarisation for synchronous parsing of semantic and syntactic dependencies. In IJCAI. pages 1562-1567.

Kristina Toutanova, Christopher D. Manning, Dan Flickinger, and Stephan Oepen. 2005. Stochastic HPSG parse disambiguation using the redwoods corpus. Research on Language and Computation 3(1):83-105.

Oriol Vinyals, Meire Fortunato, and Navdeep Jaitly. 2015a. Pointer networks. In Advances in Neural Information Processing Systems 28. pages 2692 2700. http://papers.nips.cc/paper/5866-pointernetworks.pdf.

Oriol Vinyals, Łukasz Kaiser, Terry Koo, Slav Petrov, Ilya Sutskever, and Geoffrey Hinton. 2015b. Grammar as a foreign language. In Advances in Neural Information Processing Systems. pages 2755-2763.

Chuan Wang, Sameer Pradhan, Xiaoman Pan, Heng Ji, and Nianwen Xue. 2016. Camr at semeval2016 task 8: An extended transition-based amr parser. In Proceedings of SemEval. pages 11731178. http://www.aclweb.org/anthology/S16-1181.

Chuan Wang, Nianwen Xue, and Sameer Pradhan. 2015a. Boosting transition-based AMR parsing with refined actions and auxiliary analyzers. In Proceedings of ACL (2). pages 857-862. http://www.aclweb.org/anthology/P15-2141.pdf.

Chuan Wang, Nianwen Xue, and Sameer Pradhan. 2015b. A transition-based algorithm for AMR parsing. In Proceedings of NAACL 2015. pages 366-375. http://aclweb.org/anthology/N/N15/N151040.pdf.

Gisle Ytrestøl. 2012. Transition-Based Parsing for Large-Scale Head-Driven Phrase Structure Grammars. Ph.D. thesis, University of Oslo.
Yi Zhang and Hans-Ulrich Krieger. 2011. Large-scale corpus-driven PCFG approximation of an HPSG. In Proceedings of the 12th international conference on parsing technologies. Association for Computational Linguistics, pages 198-208.

Yi Zhang, Stephan Oepen, and John Carroll. 2007. Efficiency in unification-based n-best parsing. In Proceedings of IWPT. pages 48-59. http://www.aclweb.org/anthology/W/W07/W072207.

Yi Zhang, Stephan Oepen, Rebecca Dridan, Dan Flickinger, and Hans-Ulrich Krieger. 2014. Robust parsing, meaning composition, and evaluation: Integrating grammar approximation, default unification, and elementary semantic dependencies. Unpublished manuscript. 